\title{
Las TICs Aplicadas en la Educación y su correlacion en el Rendimiento Académico
}

\section{Applied TICs in Education and its correlation in Academic Performance}

\author{
Jenny Maritza Rosero Lozano* \\ Universidad de Guayaquil, Ecuador. \\ jenny.roserol@ug.edu.ec
}

Fecha de recepción: 8 de agosto de 2016 — Fecha de aceptación: 16 de agosto de 2016

\begin{abstract}
Resumen-El presente estudio, buscó determinar la correlación entre la aplicación de las TIC en la educación y el rendimiento académico de la asignatura de programación en lenguajes estructurados, la misma duró 10 meses y se desarrolló con 5 docentes, 73 representantes legales y 103 estudiantes que conforman toda la población correspondiente al primer curso de bachillerato técnico de servicios, especialización aplicaciones informáticas. Se fundamenta en el constructivismo de Jean Piaget, en las técnicas centradas en la individualización de la enseñanza de la teoría del aprendizaje significativo de Ausubel, las estrategias docentes para un aprendizaje significativo de Díaz Barriga. Para medir las variables se utilizó el coeficiente de correlación de Pearson (0.66), los resultados revelaron el bajo rendimiento académico de los educandos en la asignatura. Lo que motivó a desarrollar la propuesta que fue la implementación de una Webquest diseñada en Exelearning que permite crear materiales de aprendizaje que combinan texto con elementos multimedia y no requiere internet para poder ejecutarse con el objeto de facilitar al educando el desarrollo de sus habilidades, capacidades y destrezas, el docente la puede utilizar como una estrategia didáctica ya que fortalece la participación activa y creativa de los educandos y permite al usuario adquirir conocimientos y habilidades en la elaboración de programas de computadora. El software fue probado y validado, teniendo como resultado que el $87.04 \%$ de los estudiantes alcanzaron promedios superiores a 7.00, se concluye que la aplicación de las TIC en el proceso de enseñanza ayuda a mejorar el rendimiento académico.
\end{abstract}

Palabras Clave-TIC, rendimiento académico, WebQuest.

Abstract-In the present study, we want to determinate the correlation between educational TICS application and the academic effiency in the subject of programmation in Structured languages which lasted 10 months and It was developed with 5 educators, 73 legal representatives and 103 students who belong to the services Technician bachelor, speciality in Informatic applications. It is based in the Constructivism by Jean Piaget, in the techniques focused in the personalized teaching in the significant learning theory by Díaz Barriga. The correlation coefficient by Pearson was used for measuring the different variables. The results revealed the low academic effiency of the students in the subject. It motivated the development of a propose which was a "WEBQUEST"designed in Exelearning that allows to create learning materials which combine text with multimedia elements and It does not require internet to execute the functions with the purpose of facilitating the student body development in its abilities, capacities and skills, the educator can use it as a didatic strategy because It helps to keep an active and creative participation of the students and It allows to the users to acquire knowledge and skills in the computer programms creation. The software was tested and validated by the educational community, It has as a result a big acceptance, good perspectives in its use and the improvement in the students academic effiency.

Keywords-TIC, academic improvement, WebQuest.

\section{INTRODUCCIÓN}

$\mathbf{L}$ a educación ha trascendido desde las paredes del aula escolar hasta el ciber espacio. A nivel mundial se ha producido éste cambio y nuestro país Ecuador, no es la excepción, por lo cual desde la Carta Magna se promueve el aprendizaje integral de los estudiantes mediado por los sistemas informáticos, así como consta en la Constitución de la República del Ecuador (Constitucional, 2008) en su "Art. 347, numeral 8, en el capítulo de la responsabilidad del Estado: "Incorporar las tecnologías de la información y comunicación en el proceso educativo y propiciar el enlace de la enseñanza con las actividades productivas o sociales"

Las TIC desempeñan un papel importante en el proceso de aprendizaje por facilidad de manejo, ayudan a superar dificultades espacio-temporales; su variedad de herramientas constituyen el soporte apropiado para una comprensión pro-

\footnotetext{
*Licenciada en Ciencias de la Educación Especialización Informática Magister en Educación Informática.
}

funda de los contenidos curriculares; brindan utilidades que facilitan el empleo de estrategias de aprendizaje, para lograr el desarrollo de habilidades, capacidades y destrezas, posibilitando y potencializando las competencias en los estudiantes; ofrecen gran ayuda en la planificación, en la aplicación de estrategias de enseñanza que promueven en los estudiantes la capacidad de aprender a aprender, la autonomía, el razonamiento, la creatividad, el pensamiento crítico reflexivo (Cooperberg, 2002).

Además son un medio para transmitir conocimientos, los software basados en las TIC pueden convertirse en poderosas herramientas, que promueven ambientes de aprendizaje cooperativos donde el docente deja ser el centro del proceso y se convierte en facilitador o un mediador del aprendizaje de los contenidos curriculares (Ramírez, 2012).

Por otro lado, las estrategias de enseñanza son las técnicas, formas, que el docente utiliza de manera exhaustiva para impartir los contenidos curriculares y las estrategias de aprendizaje son todas las formas y técnicas que ha desa- 
rrollado el estudiante para entender la información nueva y concatenarla a la información anterior con el propósito de obtener el aprendizaje. Ausubel et al. (1998), manifiesta que el docente debe ser capaz de aplicar una enseñanza estratégica para que se produzca un aprendizaje significativo de los contenidos curriculares, el docente debe mejorar y enriquecer las oportunidades de aprender de los estudiantes, aplicando estrategias de enseñanza que son todas las ayudas que se otorgan al estudiante para que se apropie del conocimiento y las estrategias de aprendizaje que son todas las modificaciones o arreglos en el contenido del material a enseñar.

Las estrategias didácticas, son el conjunto de procedimientos y acciones que el docente debe aplicar para alcanzar el aprendizaje significativo de los estudiantes. Ramos Ramos (2012), manifiesta que es oportuno aplicar estrategias didácticas para facilitar la asimilación de los contenidos y mejorar el nivel académico de los estudiantes, se debe planificar en forma consciente y reflexiva las actividades para que se lleve a efecto el proceso de enseñanza - aprendizaje.

En cuanto al rendimiento académico, Figueroa Erazo (2012), manifiesta: "El aprovechamiento Académico se especifica como el producto del aprovechamiento del contenido de los programas de estudio, expresado en calificaciones dentro de una escala convencional". Este se refiere al efecto medible en forma cuantitativa que se obtiene al evaluar a los estudiantes mediante pruebas estructuradas y actividades adicionales, en el que se determina el nivel aprehendido de los conocimientos en las materias. Reyes Tejada (2003), define el rendimiento académico como la expresión de capacidades y de características psicológicas del estudiante desarrolladas y actualizadas a través del proceso de enseñanza-aprendizaje, que le posibilita obtener un nivel de funcionamiento y logros académicos a lo largo de un período, año o quimestre.

Para la investigación, el rendimiento académico es el resultado de la interiorización de los contenidos curriculares de una asignatura, obtenidos durante el proceso de enseñanza-aprendizaje; expresados mediante una escala cualitativa y cuantitativa el cual será medido de acuerdo a la escala de calificaciones proporcionada por el Ministerio de Educación, según como lo establecen los artículos 194 y 186 del Reglamento a la Ley Orgánica de Educación Intercultural (2015). En cuanto a éste, se percibe que el $31.06 \%$ de los estudiantes no alcanzan los aprendizajes requeridos sus promedios cuantitativos son inferiores a 7.00, no alcanzan los aprendizajes requeridos (NAR), motivo por el que varios estudiantes requieren recuperación y refuerzo académico durante el año lectivo y el $23.30 \%$ recurren al examen de supletorio, al examen remedial y hasta al examen de gracia para aprobar la asignatura y por ende el año escolar. En este punto, cabría preguntarse ¿La incorporación de las TIC en el proceso educativo, ayudaría a mejorar el rendimiento académico de los estudiantes en la asignatura Programación en Lenguajes Estructurados?

El principal objetivo de la investigación fue determinar la correlación entre la aplicación de estrategias de enseñanza mediadas por las Tecnologías de la Información y la Comunicación y el mejoramiento del rendimiento académico de los estudiantes y se propuso diseñar una WebQuest como herramienta didáctica.

\section{MATERiales Y Métodos}

La investigación que se realizó fue se tipo experimental, porque se diseñó, desarrollo e implementó una herramienta didáctica mediada por TIC, la Webquest se utilizó como apoyo de estudio de la asignatura Programación en Lenguajes Estructurados cuyos contenidos se imparten en el primer curso del Bachillerato Técnico de Servicios.

Al mismo tiempo, se aplicó la investigación correlacional, la misma que permitió identificar la relación entre la aplicación de las TIC en el proceso de enseñanza y el rendimiento académico, para su efecto la variable dependiente "Rendimiento académico" fue medida en dos grupos de estudiantes de diferentes secciones, solo al grupo 2 se le aplicó el software.

El estudio se realizó en el Colegio Fiscal "Provincia de Tungurahua", durante el periodo lectivo 2014-2015, el universo objeto de estudio fue de 95 directivos y docentes, 2180 representantes legales y 2211 estudiantes que conforman la comunidad educativa y la muestra fue toda la población de estudiantes pertenecientes al primer curso de bachillerato con especialización aplicaciones informáticas, 103 estudiantes, 5 directivos y docentes y 73 padres de familia (Tabla 1).

Tabla 1. Población y Muestra.

\begin{tabular}{|l|r|r|}
\hline & POBLACIÓN & MUESTRA \\
\hline AUTORIDADES Y DOCENTES & 95 & 5 \\
\hline ESTUDIANTES & 2211 & 103 \\
\hline REPRESENTANTES LEGALES & 2180 & 73 \\
\hline TOTAL & 4486 & 181 \\
\hline
\end{tabular}

Fuente: Colegio Fiscal Provincia de Tungurahua.

Entre las técnicas de recopilación de información se aplicó las entrevistas y las encuestas, para la codificación de las respuestas se utilizaron la escala de Likert, y para la medición de la variable se aplicó el coeficiente de correlación de Pearson.

\section{DESCRIPCIÓN DE LA APLICACIÓN WEBQUEST - TIC}

Para el diseño y desarrollo de la WebQuest se utilizó el software eXeLearning que es un programa para la creación de actividades educativas es de código abierto, de sencillo manejo que incorpora una gran cantidad de herramientas y no se requiere tener conocimientos de programación para su uso (Bernal, 2014). Permite al usuario interactuar con una guía didáctica cuidadosamente elaborada por el docente, donde prepara recursos especialmente procedentes de internet, permite desarrollar las habilidades y destrezas de los educandos.

Mediante la herramienta digital se proveyó al estudiante información sobre la asignatura, los objetivos, los contenidos; se indicó la organización del curso, las técnicas de aprendizaje y las tareas; se describió los pasos que el estudiante debe seguir para realizar los ejercicios propuestos en la tarea y se proporcionó una serie de videos algunos elaborados por la docente y otros subidos al internet por otros autores los mismos que sirvieron de guía para que cada grupo de estudiantes pueda resolver los ejercicios propuestos; se proporcionó una prueba objetiva de los contenidos curriculares, elaborados cuidadosamente pos la docente aplicando los idevice que proporciona la herramienta digital. 


\section{RESULTADOS Y DisCUSIÓN}

Entre las preguntas relevantes aplicadas a los estudiantes y a los padres de familia se obtuvieron los resultados:

Los estudiantes y representantes legales manifiestan que una de las asignaturas más difíciles de aprender es programación en lenguajes estructurados; indican que casi nunca obtienen calificaciones superiores a 7.00 y a menudo necesitan ayuda para resolver los ejercicios; que el docente organiza grupos de estudio para mejorar el rendimiento académico sin embrago no obtiene mayores resultados.

Así mismo, opinan que les llama la atención los avances tecnológicos y es beneficioso el uso de la tecnología dentro y fuera del aula de clases, que la implementación de una Webquest podría ayudar a mejorar su rendimiento académico.

En cuanto a la aplicación de la WebQuest, Los resultados obtenidos de la correlación de la aplicación de las TIC con el rendimiento académico, evidencia: los estudiantes que recibieron las clases utilizando el software, tuvieron un incremento en sus calificaciones, la aplicación de Pearson indica que existe una correlación positiva de 0.66 (Figuras 1 y 2).

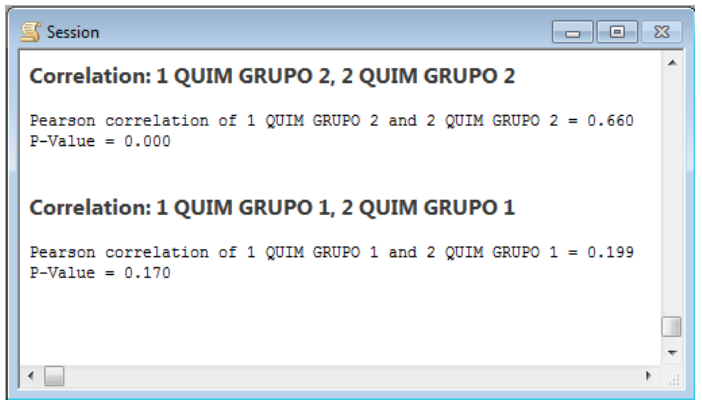

Figura 1. Correlación de Pearson.

Fuente: Elaboración propia.

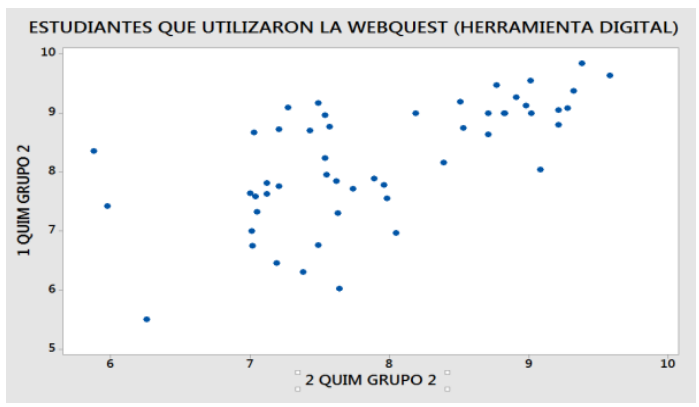

Figura 2. Rendimiento Académico.

Fuente: Elaboración propia.

Por su parte la información obtenida de las actas de calificaciones revelan: el grupo donde se aplicó el software obtuvieron el $87.04 \%$ de los estudiantes aprobaron directamente el año escolar en la asignatura, el $12.96 \%$ tubo que rendir examen supletorio y no hubo examen remedial, a diferencia del grupo donde no se aplicó el software tuvieron promedios inferiores, así el $65.31 \%$ aprobaron directamente, el $30.61 \%$ se presentaron a rendir examen supletorio y hasta hubo el $4.08 \%$ de estudiantes que tuvieron que rendir el examen remedial, para poder aprobar la asignatura (Figura 3). En resumen solo el $12.96 \%$ de estudiantes del grupo 2, obtuvieron un promedio inferior a 7.00 puntos, a diferencia del $34.69 \%$ perteneciente al grupo 1, cuyo promedio final no alcanzó lo mínimo requerido para aprobar en forma directa el año escolar.

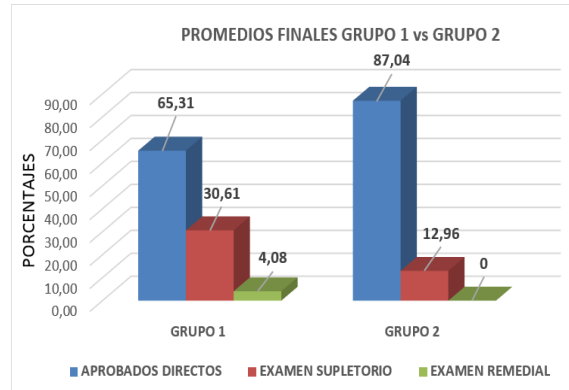

Figura 3. Promedios Finales.

Fuente: Elaboración propia.

Así mismo, estos resultados coinciden con los resultados de otras investigaciones (Peñaloza and Alba, 2014; Ariza and de la Serna, 2000) que precisamente indagan la relación que existe entre las estrategias de aprendizaje con el rendimiento académico de los estudiantes. Estos resultados pueden corroborarse con los obtenidos en las encuestas dirigidas a los docentes, estudiantes y representantes legales, donde indican que siempre las herramientas didácticas digitales son de gran apoyo dentro y fuera del aula de clases, que son de gran utilidad al docente para mejorar el rendimiento académico de los estudiantes.

\section{CONCLUSIÓN}

En conclusión, los estudiantes que utilizaron las TIC como material de apoyo en el proceso de enseñanza mejoraron su rendimiento académico reflejados en mayores calificaciones en el acta final del periodo escolar; la experiencia ha sido positiva. El resultado muestra que el diseño e implementación de la WebQuest, en el proceso de enseñanza, ayuda a mejorar el rendimiento académico de los estudiantes en la asignatura Programación en Lenguajes Estructurados. La aplicación de las TIC en el ámbito educativo es de gran importancia, permite al usuario interactuar con una guía didáctica cuidadosamente elaborada por el docente, donde planifica la clase seleccionando la mejor estrategia a utilizar en cada uno de los momentos del aprendizaje, la aplicación de herramientas didácticas digitales como material de apoyo al docente pueden ser aplicados en cualquier nivel de estudios.

\section{REFERENCIAS BIBLIOGRÁFICAS}

Ariza, J. and de la Serna, M. (2000). Nuevas tecnologías de la información y de la comunicación aplicadas a la educación. Biblioteca de Educación. Aljibe.

Ausubel, D., Novak, J., and Hanesian, H. (1998). Psicología educativa, un punto de vista cognoscitivo. primera edición en español (1983) méxico: Editorial trillas. Décimoprimera reimpresión.[Links]. 
Constitucional, T. (2008). Constitución de la república del ecuador. Quito-Ecuador: Registro Oficial, 449:20-10.

Cooperberg, A. F. (2002). Las herramientas que facilitan la comunicación y el proceso de enseñanza-aprendizaje en los entornos de educación a distancia. Revista de educación a distancia, (3).

Figueroa Erazo, D. M. (2012). Psicología de la adolescencia : desarrollo del programa oficial de segundo año de bachillerato - Figueroa Erazo, Daysi Miriam - Librero Online.

Peñaloza, H. A. B. and Alba, A. L. (2014). La influencia de las tic en el desempeño académico: evidencia de la prueba pirls en colombia 2011. Revista Academia y Virtualidad, 7(2):15-26.

Ramírez, M. (2012). Modelos y estrategias de enseñanza para ambientes innovadores. Monterrey, México: Editorial Digital Tecnológico de Monterrey.

Ramos Ramos, K. V. (2012). Estrategias didácticas para mejorar el nivel académico de los estudiantes de la escuela fiscal frank vargas pazzos del cantón salinas en el año 20122013.

Reyes Tejada, Y. N. (2003). Relación entre el rendimiento académico, la ansiedad ante los exámenes, los rasgos de personalidad, el autoconcepto y la asertividad en estudiantes del primer año de psicología de la unmsm. 